\title{
FINITE NILPOTENT CHARACTERISTIC NONVERBAL GROUPS
}

\author{
BY \\ ORIN CHEIN
}

\begin{abstract}
In this paper, we study nilpotent groups which are quotient groups of finitely generated free groups with respect to characteristic but nonverbal subgroups. We show that there are no abelian groups of the type in question. We also show that all such groups of nilpotence class 2 or 3 are finite and have minimal sets of two generators. In fact, formal presentations for all such groups are given.

The direct product of two finite CNV groups (as the groups in question will be called) which have minimal sets of generators of the same size is shown to again be a CNV group, provided that the orders of the original two groups are relatively prime. Conversely, if a finite CNV group is a direct product of groups of relatively prime orders, then at least one of these direct factors is a CNV group. Several other related results are also obtained.
\end{abstract}

1. Introduction and summary. It is well known that every verbal subgroup of any group is fully invariant, that every fully invariant subgroup is characteristic, and that every characteristic subgroup is normal. It is also known that every fully invariant subgroup of a free group is verbal [6, Theorem 12.34, p. 5]. It is natural to ask whether every characteristic subgroup of a free group is fully invariant and hence verbal. The answer is no, but very few examples to the contrary are known. B. H. Neumann [5, Theorem 2.3, p. 145] conjectured that characteristic subgroups of free groups of infinite rank are fully invariant. D. E. Cohen [1, p. 445] proved a corresponding conjecture for free abelian-by-nilpotent groups of countable rank. As far as finitely generated free groups are concerned, we are aware of only three known examples of characteristic but not fully invariant subgroups: The classic example is the subgroup of a free group of rank 2, which gives rise to the quaternions as its quotient group [5, p. 146]; Neumann [5, p. 150] also mentions an example due to MacDonald - the characteristic closure of $x_{1}^{8}$ in the free group of rank three; Cohen [1, p. 448] gives an example of a characteristic but not fully invariant subgroup of a free metabelian group. As far as we know, only the first of these examples gives rise to a finite quotient group.

Presented to the Society, February 12, 1969; received by the editors August 22, 1969.

AMS Subject Classifications. Primary 2027, 2054; Secondary 2008, 2010, 2027, (2025, 2040?).

Key Words and Phrases. Free group, characteristic subgroup, fully invariant subgroup, verbal subgroup, quaternions, nilpotent group, abelian group, finite group, minimal set of generators, direct product, direct factors, quotient group, Nielsen transformations, commutator, relation, presentation, Witt-Hall identities, $\boldsymbol{P}$-group, nilpotence class.

Copyright (C) 1970, American Mathematical Society 
In the present paper, we will study finite nilpotent CNV groups, where a CNV group is a group which is the quotient of some free group with respect to a characteristic but not verbal subgroup. In particular, we will show the following:

1. An abelian group is not a CNV group.

2. A finitely generated nilpotent CNV group of class 2 or 3 must be finite, must have a minimal set of two generators, and must have a quotient group isomorphic with the quaternions. Presentations for such groups are given.

3. The direct product of finite CNV groups of pairwise relatively prime orders is a finite CNV group; and, conversely, if the direct factors of a finite CNV group are of pairwise relatively prime order, then at least one of them is a finite CNV group.

The author of this paper would like to express his appreciation to Wilhelm Magnus for the discussions he has had with him in connection with this problem.

2. Notation and preliminaries. For the purposes of word economy, we will call a group $G$ which is isomorphic to the quotient of a free group with respect to some characteristic subgroup a $C$-group. If $G \simeq F / C$ and $y_{1}, \ldots, y_{n}$ generate $F$, then $x_{1}, \ldots, x_{n}$ generate $G$, where $x_{i}$ is the image of the coset $y_{i}+C$ under the isomorphism. Performing any Nielsen transformation on the $x_{i}$ 's must give rise to an automorphism of $G$, since the corresponding Nielsen transformation on the $y_{i}$ 's gives rise to an automorphism of $F$, and $C$ is characteristic. Conversely, if a group $G$ has a set of generators with respect to which all Nielsen transformations induce automorphisms, then the group is the quotient group of a free group with respect to a characteristic subgroup. Thus a group $G$ will be called a $C$-group if it has some set of generators with respect to which all Nielsen transformations induce automorphisms of $G$.

Note the signficance of the words "some set of generators." It is known that there are groups which possess two different minimal sets of generators such that neither can be derived by performing a Nielsen transformation on the other-not even by first returning to the underlying free group [9, Theorem 1, p. 228]. Thus it is possible that a group be a $C$-group with respect to one set of generators, but not with respect to another. Whether or not there is a group in which this can actually happen is still an open question. It is also unknown whether a group can be a $C$-group with respect to a set of generators of nonminimal size.

A group $G$, which is the quotient group of some free group with respect to some verbal subgroup will be called a $V$-group. Here, clearly, the choice of a presentation for $G$ plays no role.

A group which is a $C$-group but not a $V$-group will be referred to as a characteristic-nonverbal group or a CNV group for short.

The notation $(a, b)$ will be used to denote the commutator $a^{-1} b^{-1} a b$ of $a$ and $b$. The following procedure will be used constantly throughout this paper.

Suppose $G$ is generated by $x_{1}, \ldots, x_{n}$ and we have relations

$$
R_{j}\left(x_{1}, \ldots, x_{n}\right)=1, \quad j=1, \ldots, m .
$$


Suppose $\mu$ is a Nielsen transformation taking $x_{1}, \ldots, x_{n}$ into $y_{1}, y_{2}, \ldots, y_{n}$. Then by "performing the Nielsen transformation $\mu$ on $R_{i}\left(x_{1}, \ldots, x_{n}\right)$ and cancelling," we will mean considering

$$
R_{i}\left(y_{1}, \ldots, y_{n}\right)\left[R_{i}\left(x_{1}, \ldots, x_{n}\right)\right]^{-1}=1
$$

possibly using the relations

to simplify.

$$
R_{j}\left(x_{1}, \ldots, x_{n}\right)=1, \quad j=1, \ldots, m
$$

For example, if $G=\left\langle x, z \mid x^{2} z^{2}=(x, z)=1\right\rangle$, and $\mu$ is the Nielsen transformation leaving $z$ fixed and taking $x$ into $x z$, then performing $\mu$ on $x^{2} z^{2}=1$ and cancelling gives $(x z)^{2} z^{2}\left(z^{-2} x^{-2}\right)=1$ or, on simplifying [using $(x, z)=1$ ], $z^{2}=1$.

Sometimes we will just " perform the Nielsen transformation $\mu$ on $R_{i}\left(x_{1}, \ldots, x_{n}\right)$ " without cancelling, meaning we will consider $R_{i}\left(y_{1}, \ldots, y_{n}\right)=1$.

If $G$ is a $C$-group with respect to the set of generators $x_{1}, \ldots, x_{n}$ and $R\left(x_{1}, \ldots, x_{n}\right)$ $=1$ is any relation which holds in $G$, then performing any Nielsen transformation on $R$, with or without cancellation, must result in a relation which holds in $G$. Also, performing a Nielsen transformation on this new relation gives yet another relation and so on.

This is the technique we will use.

The group of Nielsen transformations on the set $\left\{x_{1}, \ldots, x_{n}\right\}$ is generated by the following particular Nielsen transformations [7]:

and

$$
\begin{aligned}
\pi_{i j}: & x_{i} \rightarrow x_{j}, \\
& x_{j} \rightarrow x_{i}, \\
& x_{k} \rightarrow x_{k}, \quad k \neq i, j, \\
\Omega_{i}: & x_{i} \rightarrow x_{i}^{-1}, \\
& x_{j} \rightarrow x_{j}, \quad j \neq i,
\end{aligned}
$$

$$
\begin{aligned}
\delta_{i j}: x_{i} & \rightarrow x_{i} x_{j} \\
x_{k} & \rightarrow x_{k}, \quad k \neq i,
\end{aligned}
$$

for $1 \leqq i<j \leqq n, 1 \leqq k \leqq n$. Therefore, to see if a group $G$ is a $C$-group with respect to a particular set of generators, it is enough to see that each of the elementary Nielsen transformations given above, when applied to the set of generators in question, induces an automorphism of $G$.

If $M$ and $N$ are Nielsen transformations, then $M N$ will denote the Nielsen transformation of first applying $N$ and then applying $M$ to the result.

Finally, in the course of simplifying relations, we will frequently make use of Hall's identities [2, p. 43]:

$$
\begin{aligned}
& (a, b) \cdot(b, a)=1 \\
& (a, b \cdot c)=(a, c) \cdot(a, b) \cdot(a, b, c) \\
& (a \cdot b, c)=(a, c) \cdot(a, c, b) \cdot(b, c)
\end{aligned}
$$


and of a weak form of a consequence of Witt's identity [4, Theorem 5.3, p. 293]namely that if $G_{k}$ represents the $k$ th group of the lower central series of $G$, and if $a \in G_{r}, b \in G_{s}$ and $c \in G_{t}$, then

$$
(a, b, c) \cdot(b, c, a) \cdot(c, a, b) \in G_{r+s+t+1} .
$$

\section{Direct products.}

LEMMA 1. If $K$ is a finite $C N V$ group with respect to some set of $n$ generators, and if $H$ is a $C$-group with respect to a set of $n$ generators, and if $(|K|,|H|)=1$ (i.e., if the order of $K$ and the order of $H$ are relatively prime), then $K \times H$ is a CNV group with respect to a set of $n$ generators.

Proof. Suppose $\left\{k_{1}, \ldots, k_{n}\right\}$ and $\left\{h_{1}, \ldots, h_{n}\right\}$ are the sets of generators in question, for $K$ and $H$ respectively. Then $\left\{k_{1} h_{1}, \ldots, k_{n} h_{n}\right\}$ generates $K \times H$. If $\mu$ is a Nielsen transformation such that

$$
\mu\left(\left\{x_{1}, \ldots, x_{n}\right\}\right)=\left\{\mu_{1}\left(x_{1}, \ldots, x_{n}\right), \ldots, \mu_{n}\left(x_{1}, \ldots, x_{n}\right)\right\}
$$

then

$\mu\left(\left\{k_{1} h_{1}, \ldots, k_{n} h_{n}\right\}\right)=\left\{\mu_{1}\left(k_{1}, \ldots, k_{n}\right) \mu_{1}\left(h_{1}, \ldots, h_{n}\right), \ldots, \mu_{n}\left(k_{1}, \ldots, k_{n}\right) \mu_{n}\left(h_{1}, \ldots, h_{n}\right)\right\}$

since $k_{i}$ and $h_{j}$ commute. But then this is an automorphism of $K \times H$, since $\mu\left(\left\{k_{1}, \ldots, k_{n}\right\}\right)$ is an automorphism of $K$ and $\mu\left(\left\{h_{1}, \ldots, h_{n}\right\}\right)$ is an automorphism of $H$ and since

$$
\begin{aligned}
& g p\left\langle\mu_{1}\left(k_{1}, \ldots, k_{n}\right) \mu_{1}\left(h_{1}, \ldots, h_{n}\right), \ldots, \mu_{n}\left(k_{1}, \ldots, k_{n}\right) \mu_{n}\left(h_{1}, \ldots, h_{n}\right)\right\rangle \\
& \quad=g p\left\langle\mu_{1}\left(k_{1}, \ldots, k_{n}\right), \ldots, \mu_{n}\left(k_{1}, \ldots, k_{n}\right)\right\rangle \times g p\left\langle\mu_{1}\left(h_{1}, \ldots, h_{n}\right), \ldots, \mu_{n}\left(h_{1}, \ldots, h_{n}\right)\right\rangle .
\end{aligned}
$$

Therefore, $K \times H$ is a $C$-group.

On the other hand, since $K$ is nonverbal, there exists a relation $R\left(k_{1}, \ldots, k_{n}\right)=1$ in $K$, and elements $u_{i}=w_{i}\left(k_{1}, \ldots, k_{n}\right), i=1, \ldots, n$ in $K$, such that $R\left(u_{1}, \ldots, u_{n}\right) \neq 1$. Consider the relation in $K \times H$ given by

$$
\begin{aligned}
\bar{R}\left(k_{1} h_{1}, \ldots, k_{n} h_{n}\right) & \equiv\left[R\left(k_{1} h_{1}, \ldots, k_{n} h_{n}\right)\right]^{|H|} \\
& =\left[R\left(k_{1}, \ldots, k_{n}\right)\right]^{|H|}\left[R\left(y_{1}, \ldots, y_{n}\right)\right]^{|H|}=1 .
\end{aligned}
$$

But

$$
\begin{aligned}
& \bar{R}\left(w_{i}\left(k_{1} h_{1}, \ldots, k_{n} h_{n}\right), \ldots, w_{n}\left(k_{1} h_{1}, \ldots, k_{n} h_{n}\right)\right) \\
& \quad=R\left(u_{1}, \ldots, u_{n}\right)^{|H|} R\left(w_{i}\left(h_{1}, \ldots, h_{n}\right), \ldots, w_{n}\left(h_{1}, \ldots, h_{n}\right)\right)^{|H|}=R\left(u_{1}, \ldots, u_{n}\right)^{|H|} \neq 1
\end{aligned}
$$

since $(|H|,|K|)=1$. Therefore, $K \times H$ is not a $V$-group. Q.E.D.

This lemma leads us to new CNV groups. In particular, since $B_{3}$ (the Burnside group of exponent 3) is a two generator $C$-group, $Q \times B_{3}$ is a CNV group. It has the following presentation

$$
Q \times B_{3}=\left\langle x, y \mid x^{12}=(x, y, x)=(x, y, y)=1 ; x^{6}=y^{6}=(x, y)^{3}\right\rangle .
$$


We will show that any two generator CNV group must have a presentation of a similar nature.

LeMma 2. If $G=H \times K$ is a finite $C$-group with respect to a set of $n$ generators, and if $(|H|,|K|)=1$, then $H$ and $K$ are both $C$-groups, each with respect to sets of $n$ generators.

Proof. Suppose $\left\{g_{1}, \ldots, g_{n}\right\}$ is the set of generators of $G$ in question. Since $G=H \times K, g_{i}=h_{i} k_{i}, i=1, \ldots, n$ and $\left\{h_{1}, \ldots, h_{n}\right\},\left\{k_{1}, \ldots, k_{n}\right\}$ are sets of generators for $H$ and $K$ respectively. Suppose $R\left(h_{1}, \ldots, h_{n}\right)=1$ is a relation holding in $H$, and suppose $\mu$ is any Nielsen transformation given as above by (3.1). Then, to show that $H$ is a $C$-group, it is enough to show that

$$
R\left(\mu_{1}\left(h_{1}, \ldots, h_{n}\right), \ldots, \mu_{n}\left(h_{1}, \ldots, h_{n}\right)\right)=1 .
$$

Consider the relation in $G$ given by

$$
\bar{R}\left(g_{1}, \ldots, g_{n}\right) \equiv\left[R\left(g_{1}, \ldots, g_{n}\right)\right]^{|K|}=\left[R\left(h_{1}, \ldots, h_{n}\right)\right]^{|K|}\left[R\left(k_{1}, \ldots, k_{n}\right)\right]^{|K|}=1 .
$$

Since $G$ is a $C$-group

But this is just

$$
\bar{R}\left(\mu_{1}\left(g_{1}, \ldots, g_{n}\right), \ldots, \mu_{n}\left(g_{1}, \ldots, g_{n}\right)\right)=1 .
$$

$$
\begin{aligned}
& \bar{R}\left(\mu_{1}\left(h_{1}, \ldots, h_{n}\right) \mu_{1}\left(k_{1}, \ldots, k_{n}\right), \ldots, \mu_{n}\left(h_{1}, \ldots, h_{n}\right) \mu_{n}\left(k_{1}, \ldots, k_{n}\right)\right) \\
& =\left[R\left(\mu_{1}\left(h_{1}, \ldots, h_{n}\right), \ldots, \mu_{n}\left(h_{1}, \ldots, h_{n}\right)\right)\right]^{|K|}\left[R\left(\mu_{1}\left(k_{1}, \ldots, k_{n}\right), \ldots, \mu_{n}\left(k_{1}, \ldots, k_{n}\right)\right)\right]^{|K|} \\
& =\left[R\left(\mu_{1}\left(h_{1}, \ldots, h_{n}\right), \ldots, \mu_{n}\left(h_{1}, \ldots, h_{n}\right)\right)\right]^{|K|} .
\end{aligned}
$$

But, since $(|H|,|K|)=1$, this can $=1$ only if

$$
R\left(\mu_{1}\left(h_{1}, \ldots, h_{n}\right), \ldots, \mu_{n}\left(h_{1}, \ldots, h_{n}\right)\right)=1
$$

which is what had to be proved. Therefore, $H$ is a $C$-group. The proof for $K$ is analogous.

Lemma 3. If $(|H|,|K|)=1$, and $H$ and $K$ are finite $V$-groups, then so is $H \times K$.

The proof of Lemma 3 will be omitted, as it involves only a simple application of the techniques used in the proofs of Lemmas 1 and 2.

As a direct consequence of Lemmas 2 and 3, we have the following:

LEMMA 4. If $G=H \times K$ is a finite $C N V$ group, and $(|H|,|K|)=1$, then at least one of $H$ and $K$ is a finite $C N V$ group.

Applying Lemmas 1 and 2, we get the converse of Lemma 3, namely

Lemma 5. If $G=H \times K$ is a finite $V$-group and $(|H|,|K|)=1$, then $H$ and $K$ are both finite $V$-groups.

Lemmas 4 and 5 tell us that, in order to find all nilpotent finite CNV groups, it is enough to find all finite $P$-groups which are $\mathrm{CNV}$ groups or $V$-groups. 


\section{The abelian case.}

THEOREM 1. There are no abelian CNV groups.

Proof. Levi [3, Satz 2, p. 91] proved that every characteristic subgroup of a free group $F$ which contains the commutator subgroup $F^{\prime}$ is the subgroup of $F$ generated by the commutators and the $m$ th powers of all elements of $F$, for some $m$. Such a subgroup is verbal. Therefore, since any abelian $C$-group is the quotient group of a free group with respect to a characteristic subgroup which contains $F^{\prime}$, every abelian $C$-group is a $V$-group.

\section{Nilpotence class 2 .}

LEMMA 6. If $F$ is a free group on free generators $\left\{y_{1}, \ldots, y_{n}\right\}$, and if $\alpha(1), \ldots, \alpha(n)$ are integers whose greatest common divisor is $d$, then there exists a Nielsen transformation $\mu$ taking $\left\{y_{i}\right\}$ into a set $\left\{z_{i}\right\}$ of free generators of $F$, such that, if $z_{1}$ is written as a word in the $y_{i}$, then the exponent sum of $y_{i}$ in this word is $\alpha(i) / d$, for all $i=1, \ldots, n$.

Proof. By induction, this easily reduces to the case $n=2$, which can be proven by abelianizing and using Nielsen's results on the two dimensional modular group [8, p. 4].

THEOREM 2. If $G$ is a group of nilpotence class 2 , which is a $C$-group with respect to a set of generators $\left\{x_{1}, \ldots, x_{n}\right\}$, where $x_{1}$ is of finite order $m$, then $G$ is of exponent $\mathrm{m}$.

Proof. Since $G$ is a $C$-group, ord $x_{i}=\operatorname{ord} x_{j}$ for all $i, j=1, \ldots, n$. Let $p=\operatorname{ord}\left(x_{1}, x_{2}\right)$. Again, since $G$ is a $C$-group, $p=$ ord $\left(x_{i}, x_{j}\right)$ for all $i \neq j$. Consider $\left(x_{i}^{m}, x_{j}\right)$. Since $G$ is nilpotent of class 2 , this is equal to $\left(x_{i}, x_{j}\right)^{m}$. But, since $x_{i}^{m}=1$, it also is equal to 1 . Therefore $p \mid m$. Also, since $G$ is nilpotent of class 2 , the commutator subgroup $G^{\prime}$ is generated by the set $\left\{\left(x_{i}, x_{j}\right)\right\}$ and hence any element of $G^{\prime}$ is of order which divides $p$ and hence which divides $m$.

Now consider any element $x$ of $G$. It can be written in the form

$$
x=x_{1}^{\alpha(1)} x_{2}^{\alpha(2)} \cdots x_{n}^{\alpha(n)} c,
$$

where $c \in G^{\prime}$, and where $0 \leqq \alpha(i)<m$ for all $i$. Let $d$ be the greatest common divisor of $\alpha(1), \ldots, \alpha(n)$, and let $\alpha^{*}(i)=\alpha(i) / d$. Consider the free group $F$ on $n$ generators $y_{1}, \ldots, y_{n}$. Let $\mu$ be the Nielsen transformation given in Lemma 6 . Then

$$
z_{1}=y_{1}^{\alpha^{*}(1)} y_{2}^{\alpha^{*}(2)} \cdots y_{n}^{\alpha^{*}(n)} w, \quad \text { where } w \text { is in } F^{\prime} .
$$

Let $\theta$ be the homomorphism of $F$ onto $G$ defined by $\theta\left(y_{i}\right)=x_{i}$. Let $v=\theta\left(z_{1}\right)$. Then the Nielsen transformation $\mu$ maps $x_{1}$ into $v$. Therefore, since $G$ is a $C$-group with respect to the $x_{i}$ 's, $v^{m}=1$.

From the way that $v$ was defined, $x=v^{d} c^{\prime}$ where $c^{\prime}$ is in $G^{\prime}$. Therefore,

$$
x^{m}=\left(v^{d} c^{\prime}\right)^{m}=v^{d m} c^{\prime m}=\left(v^{m}\right)^{d}\left(c^{\prime}\right)^{m}=1 .
$$

But this is true for an arbitrary $x \in G$. Q.E.D. 
If there are no relations in $G$ which are not a consequence of the nilpotence or of the fact that $G$ is of exponent $m$, then $G$ is a $V$-group. If there is any other relation, it can be expressed in the form

$$
x_{1}^{\alpha(1)} x_{2}^{\alpha(2)} \cdots x_{n}^{\alpha(n)}=c,
$$

where $c \in G^{\prime}$ and $0 \leqq \alpha(i)<m$ for all $i=1, \ldots, n$. (Since $G$ is not abelian, $n \geqq 2$.)

Of all such relations, consider one in which $\alpha(n)$ is minimal.

Claim. In such a relation $\alpha(n)=0$.

Proof. If $\alpha(n) \neq 0$, then, by the division algorithm, there exist $q, r$ such that $\alpha(1)=q \alpha(n)+r$, where $0 \leqq r<\alpha(n)$. Performing the Nielsen transformation $\delta_{n 1}^{-q} \pi_{1 n}$ on the relation and cancelling, we get a new relation in which the exponent of $x_{n}$ is $r$, which is smaller than $\alpha(n)$. This is a contradiction, since $\alpha(n)$ was chosen to be minimal, and so the claim is proven.

Repeating this procedure with $\alpha(n-1), \alpha(n-2), \ldots$, we eventually arrive at a relation of the form

$$
x_{1}^{\alpha}=c=\prod_{i<j}\left(x_{i}, x_{j}\right)^{\beta(i, j)}
$$

where $0 \leqq \beta(i, j)<p$ for all $i, j=1, \ldots, n$, and $0 \leqq \alpha<m$. Since we are assuming this is a nontrivial relation, and since $\alpha<m$, there exists a nonzero $\beta(i, j)$, say $\beta\left(i_{0}, j_{0}\right) \neq 0$. Since $i_{0}<j_{0}, j_{0} \neq 1$.

Case 1. If $n \geqq 3$, we can find $k_{0} \neq j_{0}, i_{0}$. Performing $\delta_{j_{0}, k_{0}}$ on (5.1) and cancelling (using the Witt-Hall identities and the fact that $G$ is nilpotent) gives the relation

$$
\prod_{i \neq j_{0}}\left(x_{i}, x_{k_{0}}\right)^{\beta\left(i, j_{0}\right)}=1 \text {. }
$$

Now $i_{0} \neq j_{0}, k_{0}$, so performing $\delta_{i_{0}, j_{0}}$ on this relation and cancelling gives

$$
\left(x_{j_{0}}, x_{k_{0}}\right)^{\beta\left(i_{0}, j_{0}\right)}=1 .
$$

But $0<\beta\left(i_{0}, j_{0}\right)<p$, so this is impossible. Therefore, we have proven the following theorem:

THEOREM 3. There are no groups of nilpotence class 2, which are CNV groups with respect to any set of more than 2 generators.

Actually, we have only proven this for finite groups, but the proof of the fact that there can be no relations of the form

$$
x_{1}^{\alpha(1)} \cdots x_{n}^{\alpha(n)}=c
$$

did not really depend on the finiteness of $G$, so the theorem holds.

Case 2. If $n=2$, then relation (5.1) can be written in the form

$$
x^{\alpha}=(x, y)^{\beta},
$$


where $0<\alpha<m$ and $0<\beta<p$. Performing $\Omega_{2}$, we get

$$
x^{\alpha}=(x, y)^{-\beta} \text {. }
$$

Combining (5.2) and (5.3), we get

$$
x^{2 \alpha}=1=(x, y)^{2 \beta}
$$

Therefore, $m=2 \alpha$ and $p=2 \beta$. Also, interchanging $x$ and $y$ in (5.2) gives

$$
y^{\alpha}=(x, y)^{-\beta}=(x, y)^{\beta} .
$$

Performing $\delta_{12}$ on (5.2) and cancelling, using the nilpotence, results in

$$
y^{\alpha}(x, y)^{\alpha(\alpha-1) / 2}=1 .
$$

But $y^{\alpha} \neq 1$, so $(x, y)^{\alpha(\alpha-1) / 2} \neq 1$, so $p$ does not divide $\alpha(\alpha-1) / 2$.

From (5.2) and (5.5), we see that

$$
x^{\alpha}=y^{\alpha},
$$

so we have

$$
1=\left(y^{\alpha}, y\right)=\left(x^{\alpha}, y\right)=(x, y)^{\alpha},
$$

so $p$ divides $\alpha$. Let $\alpha=p q$. If $q$ is even then $2 p$ divides $\alpha$ and then $p$ divides $\alpha(\alpha-1) / 2$ which we saw cannot happen. Therefore, $q$ must be odd.

THEOREM 4. $G$ is a CNV group of nilpotence class 2 , if and only if $G$ has a presentation of the form

$$
G=\left\langle x, y \mid x^{m}=(x, y)^{p}=(x, y, x)=(x, y, y)=1 ; x^{m / 2}=y^{m / 2}=(x, y)^{p / 2}\right\rangle,
$$

where $m=2 p q$ and $q$ is odd.

Proof. If $G$ is a CNV group it must be on two generators, we know that (5.2) and (5.5) must hold, with $\alpha=m / 2$ and $\beta=p / 2$. Also, as a consequence of (5.2) and (5.5), we have

$$
x^{m / 2} y^{m / 2}=1 .
$$

If there are any other relations then, using (5.2), (5.5) and (5.8), they can be expressed in the form $x^{a} y^{b}(x, y)^{c}=1$ where $0 \leqq a<m / 2,0 \leqq b<m / 2$ and $0 \leqq c<p$. If both $a$ and $b$ are zero, then $c$ must be 0 . If either $a$ or $b$ is not zero, then going through our original argument we get a relation of type (5.2) with $\alpha<m / 2$, which we saw was impossible. Thus the only if part is proven if $G$ is finite.

Note that equation (5.4) shows that $x$ must be of finite order, and hence that $G$ must be of finite exponent by Theorem 2 and hence must be finite, since it is nilpotent. Therefore, the only if part is proven.

Suppose $G$ is of the above form. It is easily checked that if $\pi_{12}, \Omega_{1}, \Omega_{2}$ or $\delta_{12}$ acts on $\{x, y\}$ then it induces an automorphism of $G$, and so $G$ is a $C$-group. However, the mapping $x \rightarrow x^{2}, y \rightarrow y$ does not induce an automorphism, since

$$
y^{m / 2} \neq\left(x^{2}, y\right)^{p / 2}=(x, y)^{p}=1 .
$$

Therefore, $G$ is not a $V$-group. Q.E.D. 
Note also, that the group $Q \times B_{3}$ does have a presentation of the required type.

As a corollary to Theorem 4 , if $G$ is a $P$-group, then $P$ must be 2 since $m$ is even. Therefore,

Corollary. The only CNV groups of nilpotence class 2 which are P-groups are 2-groups and have presentations of the form

$$
G=\left\langle x, y \mid x^{\left(2^{n+1}\right)}=(x, y, x)=(x, y, y)=1 ; x^{\left(2^{n+1}\right)}=y^{\left(2^{n+1}\right)}=(x, y)^{\left(2^{n}\right)}\right\rangle .
$$

THEOREM 5. Every CNV group of nilpotence class 2 has the quaternion group $Q$ as a quotient group.

Proof. $G$ must have a presentation of the type given in Theorem 4. Map $G \rightarrow Q$ by $x \rightarrow u, y \rightarrow v$ where

$$
Q=g p\left\langle u, v \mid u^{4}=(u, v, u)=(u, v, v)=1 ; u^{2}=v^{2}=(u, v)\right\rangle .
$$

This is clearly a homomorphism onto $Q$.

\section{Nilpotence class 3 .}

THEOREM 6. A group $G$ of nilpotence class 3 which is a CNV group with respect to a set of generators $\left\{x_{1}, \ldots, x_{n}\right\}$, where $x_{1}$ is of finite order $m$, is of exponent $m$.

Proof. Since $\pi_{i j}$ induces an automorphism of $G$, ord $x_{i}=$ ord $x_{\jmath}$, for all $i, j$, so ord $x_{i}=m$, for all $i$. Thus we have

$$
x_{i}^{m}=1, \quad i=1, \ldots, n .
$$

Therefore, since $G$ is of nilpotence class 3,

$$
1=\left(x_{i}^{m}, x_{j}, x_{k}\right)=\left(x_{i}, x_{j}, x_{k}\right)^{m}
$$

for all $i, j, k$. Performing $\delta_{i j}$ on (6.1) and cancelling, we get

$$
1=\left(x_{i} x_{j}\right)^{m}=x_{i}^{m} x_{j}^{m}\left(x_{i}, x_{j}\right)^{m(m-1) / 2} z=\left(x_{i}, x_{j}\right)^{m(m-1) / 2} z,
$$

where $z \in G_{3}$ (the third group of the lower central series of $G$ ). Conjugating with $x_{k}$, we get

$$
\left(x_{i}, x_{j}, x_{k}\right)^{m(m-1) / 2}=1
$$

for all $i, j, k$. Taking $k=i$ and using Hall's identities, we get

$$
1=\left(x_{i}^{m}, x_{j}\right)=\left(x_{i}, x_{j}\right)^{m}\left(x_{i}, x_{j}, x_{i}\right)^{m(m-1) / 2}=\left(x_{i}, x_{j}\right)^{m}
$$

for all $i, j$. Since $G$ is of nilpotence class 3 , the commutator subgroup is abelian and is generated by the commutators $\left(x_{i}, x_{j}\right)$ and $\left(x_{i}, x_{j}, x_{k}\right)$, where $i<j, i \leqq k$ (we only need $i \leqq k$ because of Witt's identity). Therefore, by (6.2) and (6.4), if $c \in G^{\prime}$ then $c^{m}=1$. Also $G_{3}$ is generated by the commutators $\left(x_{i}, x_{j}, x_{k}\right), i<j$, $i \leqq k$, and so, by (6.3), if $c^{\prime} \in G_{3}$ then $\left(c^{\prime}\right)^{m(m-1) / 2}=1$. Finally, any element $x$ in $G$ can be expressed in the form

$$
x=x_{1}^{\alpha(1)} x_{2}^{\alpha(2)} \cdots x_{n}^{\alpha(n)} c^{\prime \prime}
$$


where $0 \leqq \alpha(i)<m$ for all $i$, and $c^{\prime \prime} \in G^{\prime}$. By the same argument used in the proof of Theorem 2, there exists a $v$ in $G$ such that $v^{m}=1$ and $x=v^{d} c$, where $d$ is the greatest common divisor of $\alpha(1), \ldots, \alpha(n)$, and $c \in G^{\prime}$. Therefore,

$$
x^{m}=\left(v^{d} c\right)^{m}=v^{d m} c^{m}\left(v^{d}, c\right)^{m(m-1) / 2} .
$$

But $v^{d m}=\left(v^{m}\right)^{d}=1$, and $c^{m}=1$, and $\left(v^{d}, c\right) \in G_{3}$, so $\left(v^{d}, c\right)^{m(m-1) / 2}=1$. Therefore $x^{m}=1$ for all $x \in G$. Q.E.D.

If we define $p$ to be the order of $\left(x_{i}, x_{j}\right), q$ to be the order of $\left(x_{i}, x_{j}, x_{i}\right)$, and $r$ to be the order of $\left(x_{i}, x_{j}, x_{k}\right)$ where $k \neq i, j$ (these are easily seen to be independent of $i, j$ and $k$ ), then (6.2), (6.3), and (6.4) show that $q|m, r| m, q \mid m(m-1) / 2$, $r \mid m(m-1) / 2$, and $p \mid m$. Performing $\Omega_{i} \pi_{i j}$ on

$$
\left(x_{i}, x_{j}, x_{i}\right)^{q}=1
$$

shows us that $q$ is also the order of $\left(x_{i}, x_{j}, x_{j}\right)$.

Must $q$ and $r$ be the same? The answer in general seems to be no, however, performing $\delta_{k i}$ on

$$
\left(x_{i}, x_{j}, x_{k}\right)^{r}=1 \text {, }
$$

we see that $q \mid r$. On the other hand, performing $\delta_{i k}$ on (6.5) and cancelling, we get

$$
\left(x_{k}, x_{j}, x_{i}\right)^{q}\left(x_{i}, x_{j}, x_{k}\right)^{q}\left(x_{k}, x_{i}, x_{k}\right)^{q}=1 \text {. }
$$

But $\left(x_{k}, x_{i}, x_{k}\right)^{q}=\left(x_{i}, x_{k}, x_{k}\right)^{-q}=1$, so

$$
\left(x_{i}, x_{j}, x_{k}\right)^{q}=\left(x_{j}, x_{k}, x_{i}\right)^{q}=\left(x_{k}, x_{i}, x_{j}\right)^{q},
$$

the last equality following by symmetry. Therefore,

$$
\left(x_{i}, x_{j}, x_{k}\right)^{3 q}=\left(x_{i}, x_{j}, x_{k}\right)^{q}\left(x_{j}, x_{k}, x_{i}\right)^{q}\left(x_{k}, x_{i}, x_{j}\right)^{q}=1
$$

by Witt's identity, since $G$ is nilpotent of class 3. Therefore, $r \mid 3 q$. But $q \mid r$ so either $r=q$ or $r=3 q$. We will show that if $G$ is a $P$-group then $P$ must be 2 and so $r$ must equal $q$ in that case.

Also, taking the commutator of $\left(x_{i}, x_{j}\right)^{p}=1$ with $x_{k}$, we see that $q \mid p$ and $r \mid p$.

Summing up, we have

$$
q|r| p|m, \quad r| m(m-1) / 2, \quad r=q \text { or } 3 q .
$$

A relation in $G$ which is not a consequence of the nilpotence, the fact that $G$ is of exponent $m$, the definitions of $m, p, q$ and $r$, (6.7) or (6.8) will be called nondeducible. Since $G$ is not a $V$-group, there must be at least one nondeducible relation in $G$. It can be expressed in the form

$$
x_{1}^{\alpha(1)} \cdots x_{n}^{\alpha(n)} c=1,
$$

where $c \in G^{\prime}$ and where $0 \leqq \alpha(i)<m$ for all $i=1, \ldots, n$. Using the same trick as in the case of nilpotence class 2 , we find that there must be a nondeducible relation of the form 


$$
x_{n}^{\alpha}=c=\prod_{i<j}\left(x_{i}, x_{j}\right)^{\beta(i, j)} \prod_{i<j ; i \leqq k}^{*}\left(x_{i}, x_{j}, x_{k}\right)^{\gamma(i, j, k)},
$$

where $0 \leqq \alpha<m, 0 \leqq \beta(i, j)<p, 0 \leqq \gamma(i, j, k)<q$ if $i=j$ or $k, 0 \leqq \gamma(i, j, k)<r$ otherwise, and $\Pi^{*}$ indicates that any reduction possible (in the case $r=3 q$ ), by virtue of the fact that $\left(x_{i}, x_{j}, x_{k}\right)^{q}\left(x_{i}, x_{k}, x_{j}\right)^{q}=1$, has been made so that, if the exponent of $\left(x_{i}, x_{j}, x_{k}\right)$ in (6.10) is a multiple of $q$, then we may assume that $\left(x_{i}, x_{k}, x_{j}\right)$ does not appear in (6.10). The fact that (6.10) is nondeducible follows from the fact that if some $\alpha(i)$ in (6.9) were nonzero then $\alpha$ would be nonzero and if all $\alpha(i)$ in (6.9) were zero, then (6.9) was already of the form (6.10). Since $x_{n}^{\alpha} \neq 1$ unless $\alpha=0$, and since (6.10) is nondeducible, at least one $\beta$ and/or at least one $\gamma$ is not zero.

Let us again consider several cases. We know $n \neq 1$, since $G$ is not abelian.

Case 1. $n=2$.

In this case, $(6.10)$ can be rewritten as

$$
y^{\alpha}(x, y)^{\beta}(x, y, x)^{y}(x, y, y)^{\delta}=1,
$$

where $x=x_{1}, y=x_{2}, 0 \leqq \alpha<m, 0 \leqq \beta<p, 0 \leqq \gamma, \delta<q$ and at least two of the exponents are nonzero. Performing $\delta_{12} \Omega_{1}^{\prime} \delta_{12} \Omega_{1}$ on (6.11) and cancelling, we get

$$
(x, y, y)^{\beta}=1 \text {, }
$$

so $q \mid \beta$. Using this fact, performing $\Omega_{1} \delta_{12}^{-1} \Omega_{1}$ on (6.11) and cancelling gives $(x, y, y)^{\gamma}$ $=1$, so $\gamma=0$. Performing $\Omega_{2}$ on (6.11) and cancelling gives

$$
y^{2 \alpha}(x, y)^{2 \beta}=1 \text { and }(x, y, y)^{2 \delta}=1 .
$$

Performing $\Omega_{1}$ on (6.11) and cancelling gives $y^{2 \alpha}=1$. Therefore,

$$
y^{2 \alpha}=(x, y)^{2 \beta}=(x, y, y)^{2 \delta}=1 .
$$

Therefore, $\alpha=0$ or $m / 2, \beta=0$ or $p / 2, \delta=0$ or $q / 2$, and at most one of them is zero. Note that this implies that, if $G$ is a $P$-group, then $P=2$, since $m, p$ or $q$ must be even.

Suppose $\alpha=0$, then neither $\beta$ nor $\delta$ can be zero. Performing $\delta_{21}$ on (6.11) and cancelling, we get

$$
(x, y, y)^{\beta}(x, y, x)^{\delta}=1 .
$$

But $q \mid \beta$, so $(x, y, y)^{\delta}=1$, which cannot be since $0<\delta<q$. Therefore, there are no nondeducible relations of the form

$$
(x, y)^{\beta}(x, y, x)^{\gamma}(x, y, y)^{\delta}=1 .
$$

The argument here does not depend upon $n$ being 2, so we can use this result for $n>2$ also. Anyway, $\alpha$ cannot be zero and hence must be $m / 2$. Performing $\pi_{12}$ on (6.11) gives

$$
x^{\alpha}(x, y)^{\beta}(x, y, x)^{\delta}=1 .
$$

Clearly, since $\alpha=m / 2$ is the minimal nonzero power of $x$ or $y$ appearing in any 
relation in $G$ (this follows from the manner in which (6.10) was obtained), and since there can be no nondeducible relations of the form (6.14), any nondeducible relation in $G$ must be a consequence of (6.11) and (6.15). Conjugating (6.15) by $y$, we get

But $q \mid \beta$, so

$$
\left(x^{\alpha}, y\right)(x, y, y)^{\beta}=1 .
$$

$$
1=\left(x^{\alpha}, y\right)=(x, y)^{\alpha}(x, y, x)^{\alpha(\alpha-1) / 2} .
$$

But this is of form (6.14) and hence must be deducible. Therefore $p \mid \alpha$ and $q \mid \alpha(\alpha-1) / 2$. Performing $\delta_{12}$ on (6.15) and cancelling with (6.11) and (6.15), we get

$$
(x, y)^{-\beta-\alpha(\alpha-1) / 2}(x, y, x)^{\alpha(\alpha-1)(\alpha-2) / 6}(x, y, y)^{\alpha(\alpha-1)(2 \alpha-1) / 6+\beta(1+\alpha)}=1 .
$$

But $q \mid \beta$ and this is of type (6.14), so $p|(\beta+\alpha(\alpha-1) / 2), q| \alpha(\alpha-1)(\alpha-2) / 6$ and $q \mid \alpha(\alpha-1)(2 \alpha-1) / 6$. Combining (6.11) and (6.15), we get

$$
x^{\alpha} y^{\alpha}(x, y, x)^{\delta}(x, y, y)^{\delta}=1 .
$$

Putting this all together, we are able to prove the following theorem.

THEOREM 7. A group of nilpotence class 3 is a CNV group with respect to a set $\{x, y\}$ of two generators if and only if $G$ has a presentation of the form

$$
\begin{aligned}
G=\langle x, y| x^{m} & =y^{m}=(x, y)^{p}=(x, y, x)^{q}=(x, y, y)^{q}=(x, y, x, x) \\
& =(x, y, x, y)=(x, y, y, y)=1 ; \\
& \left.x^{\alpha}(x, y)^{\beta}(x, y, x)^{\delta}=y^{\alpha}(x, y)^{\beta}(x, y, y)^{\delta}=1\right\rangle,
\end{aligned}
$$

where

$$
\begin{gathered}
q|p| m / 2 ; \quad \alpha=m / 2, \quad q \mid \beta, \quad \beta=0 \text { or } p / 2, \quad \delta=0 \text { or } q / 2 \\
q|\alpha(\alpha-1) / 2, \quad q| \alpha(\alpha-1)(\alpha-2) / 6, \quad p \mid(\beta+\alpha(\alpha-1) / 2)
\end{gathered}
$$

and not both $\beta$ and $\delta$ are zero.

Proof. We have already proven the only if part if $G$ is finite. If $G$ is not finite, $x$ is of infinite order and, by (6.13), $\alpha=0$ which we saw cannot happen, so the only if part is proven. Suppose $G$ is of the above type. Consider the substitution replacing $x$ by $x y$ and $y$ by 1 in (6.15). If this substitution did induce an automorphism of $G$, we would have

$$
\begin{aligned}
1 & =(x y)^{\alpha}=x^{\alpha} y^{\alpha}(x, y)^{-\alpha(\alpha-1) / 2}(x, y, x)^{\alpha(\alpha-1)(\alpha-2)}(x, y, y)^{\alpha(\alpha-1)(2 \alpha-1) / 6} \\
& =x^{\alpha} y^{\alpha}(x, y)^{-\alpha(\alpha-1) / 2}
\end{aligned}
$$

Combining with (6.16), we get

$$
(x, y)^{\alpha(\alpha-1), 2}(x, y, x)^{-\delta}(x, y, y)^{-\delta}=1 .
$$

So $\alpha(\alpha-1) / 2=0$ and $\delta=0$. But $p \mid(\beta+\alpha(\alpha-1) / 2)$, so $\beta=0$. But this is impossible, so $G$ is not a $V$-group. To show that $G$ is a $C$-group, it is enough to show that $\Omega_{1}, \Omega_{2}, \pi_{12}$ and $\delta_{12}$ all induce automorphisms of $G$ when they act on $\{x, y\}$. This is easily seen by performing these Nielsen transformations on the left-hand sides 
of the relations (6.11) and (6.15) and noting that the resulting relators are consequences of the presentation assumed for $G$. The details will be omitted. Q.E.D.

Case 2. $n \geqq 3$.

We have a nondeducible relation of form (6.10) in $G$, and not all $\beta$ 's and $\gamma$ 's are zero. If $\beta\left(i_{0}, j_{0}\right) \neq 0$, then $i_{0}<n$. Choosing $k_{0} \neq i_{0}, j_{0}$ and performing $\delta_{i_{0}, k_{0}}$ on (6.10) and cancelling, we get a relation of the type

$$
\prod_{i<j}\left(x_{i}, x_{j}\right)^{\beta(i, j)} \prod_{i<j: i \leqq k}^{*}\left(x_{i}, x_{j}, x_{k}\right)^{\gamma(i, j, k)}=1 .
$$

This relation will be nondeducible, since the term $\left(x_{k_{0}}, x_{j_{0}}\right)$ will appear with exponent $\beta\left(i_{0}, j_{0}\right)$, which is not zero. Also, from the method by which (6.18) is obtained, every term appearing involves $x_{k_{0}}$. Performing $\delta_{j_{0}, i_{0}}$ on (6.18) and cancelling results in a nondeducible relation of the form

$$
\left(x_{k_{0}}, x_{i_{0}}\right)^{\beta\left(i_{0}, j_{0}\right)}=\prod_{i<j: i \leqq k}^{*}\left(x_{i}, x_{j}, x_{k}\right)^{\gamma(i, j, k)} .
$$

Performing $\pi_{k_{0}, n-1} \pi_{i_{0}, n}$, if necessary [if $k_{0}=n-1$ or $i_{0}=n$, omit the corresponding $\pi$ ], we get a nondeducible relation of form

$$
\left(x_{n-1}, x_{n}\right)^{\beta}=\prod_{i<j ; i \leqq k}^{*}\left(x_{i}, x_{j}, x_{k}\right)^{\gamma(i, j, k)},
$$

where $0<\beta<p$, and thus not all the $\gamma$ 's can be zero. From the case $n=2$, we saw we cannot have a relation of form (6.14), therefore, there is an $i_{1}, j_{1}, k_{1}$ such that $i_{1}<n-1$ and $\gamma\left(i_{1}, j_{1}, k_{1}\right) \neq 0$. Similarly, if all $\beta(i, j)$ are zero in (6.10), then there exist $i_{1}, j_{1}, k_{1}$ such that $i_{1}<n$ and $\gamma\left(i_{1}, j_{1}, k_{1}\right) \neq 0$.

In either of the above cases we do the following. If $k_{1}=i_{1}$, then $0<\gamma\left(i_{1}, j_{1}, k_{1}\right)<q$. Performing $\delta_{i_{1}, j_{1}}$ on (6.20) (or (6.10)) and cancelling results in a relation of the form

$$
\prod_{i<j: i \leqq k}^{*}\left(x_{i}, x_{j}, x_{k}\right)^{\gamma(i, j, k)}=1 .
$$

All terms in (6.21) will involve $x_{j_{1}}$, and the term $\left(x_{i_{1}}, x_{j_{1}}, x_{j_{1}}\right)$ will appear with exponent $\gamma\left(i_{1}, j_{1}, k_{1}\right)$, since it can only result from the term $\left(x_{i_{1}}, x_{j_{1}}, x_{i_{1}}\right)$ in (6.20) (or (6.10)). Therefore (6.21) will be nondeducible, since

$$
0<\gamma\left(i_{1}, j_{1}, i_{1}\right)=\gamma\left(i_{1}, j_{1}, k_{1}\right)<q .
$$

If $k_{1}=j_{1}$, then, since $n \geqq 3$, there exists $k_{2} \neq i_{1}, j_{1}$. Performing $\delta_{i_{1}, k_{2}}$ on (6.20) (or (6.10)) again results in a nondeducible relation of the form (6.21), this time because the exponent of $\left(x_{k_{2}}, x_{j_{1}}, x_{j_{1}}\right)$ is $\gamma\left(i_{1}, j_{1}, j_{1}\right)$ which is $\gamma\left(i_{1}, j_{1}, k_{1}\right)$ which is between 0 and $q$.

If $k_{1} \neq i_{1}$ or $j_{1}$, then $0<\gamma\left(i_{1}, j_{1}, k_{1}\right)<r$. In the case $r=3 q$, if $\gamma\left(i_{1}, j_{1}, k_{1}\right)=q$ or $2 q$ then we may assume by our reduction that $\left(x_{i_{1}}, x_{k_{1}}, x_{j_{1}}\right)$ does not appear in (6.20) (or (6.10)). Performing $\Omega_{i_{1}}$ on (6.20) (or (6.10)) and cancelling results in a relation of the form (6.21) in which the exponent of $\left(x_{i_{1}}, x_{j_{1}}, x_{k_{1}}\right)$ is $-2 \gamma\left(i_{1}, j_{1}, k_{1}\right)=2 q$ or 
$4 q \neq 0$, and $\left(x_{i_{1}}, x_{k_{1}}, x_{j_{1}}\right)$ does not appear. Therefore the relation is nondeducible. Also, all terms which do appear involve $x_{i_{1}}$.

Finally, if $r=q$ or if, in (6.20) (or (6.10)), $\gamma\left(i_{1}, j_{1}, k_{1}\right.$ ) is not a multiple of $q$, performing $\delta_{i_{1}, k_{1}}$, we get a relation of type (6.21) in which the exponent of $\left(x_{k_{1}}, x_{j_{1}}, x_{k_{1}}\right)$ is $\gamma\left(i_{1}, j_{1}, k_{1}\right)$ and which therefore is nondeducible. All terms in this relation involve $x_{k_{1}}$.

Thus, if $n \geqq 3$ and $G$ has a nondeducible relation, then it has a nondeducible relation of form (6.21) in which all the terms have a common $x_{i}$ for some $i\left(j_{1}, k_{2}, i_{1}, k_{1}\right.$ respectively in the four cases just considered). Renaming the common index $i_{2}$, removing the restriction $i<j, i \leqq k$, using Witt's identity, and then applying $\pi_{i_{2} n}$, the relation can be expressed in the form

$$
\prod_{j, k}\left(x_{n}, x_{j}, x_{k}\right)^{\gamma(j, k)}=1 \text {, }
$$

where $0 \leqq \gamma(j, k)<q$ or $r$ (whichever is appropriate) and if $r=3 q$ and $\gamma(j, k)=q$ or $2 q$ then $\gamma(k, j)=0$, and not all $\gamma$ 's are 0 .

If there is a pair $j_{3}, k_{3}$, neither equal to $n-1$, for which $\gamma\left(j_{3}, k_{3}\right) \neq 0$, then performing $\delta_{j_{3}, n-1}$ on (6.22) and cancelling results in a new relation of the form (6.22) in which all the commutators appearing have $n$ and $n-1$ among their subscripts. The exponent of $\left(x_{n}, x_{n-1}, x_{k_{3}}\right)$ is $\gamma\left(j_{3}, k_{3}\right)$ and even if the term $\left(x_{n}, x_{k_{3}}, x_{n-1}\right)$ does appear, its exponent is $\gamma\left(k_{3}, j_{3}\right)$ and so the relation is nondeducible.

Thus in any case we have a nondeducible relation of the form (6.22), in which every commutator involves both $x_{n}$ and $x_{n-1}$. We know from the case $n=2$ that not all commutators can involve only $x_{n}$ and $x_{n-1}$, otherwise we would have a relation of form (6.14). Therefore, there is a commutator of the form $\left(x_{n}, x_{n-1}, x_{k_{4}}\right)^{\gamma}$ or $\left(x_{n}, x_{k_{4}}, x_{n-1}\right)^{\gamma}$ appearing in (6.22), where $k_{4} \neq n-1, n$ and $0<\gamma<r$.

If $r=q$ or $r=3 q$ but $\gamma$ is not a multiple of $q$, then performing $\delta_{k_{4}, n-1}$ and cancelling results in a nondeducible relation involving only $x_{n}$ and $x_{n-1}$, which is impossible.

If $\gamma=q$ or $2 q$ then not both of the terms $\left(x_{n}, x_{k_{4}}, x_{n-1}\right)$ and $\left(x_{n}, x_{n-1}, x_{k_{4}}\right)$ can occur, and so performing $\Omega_{k_{4}}$ and cancelling results in the relation

$$
\left(x_{n}, x_{k_{4}}, x_{n-1}\right)^{2 \gamma}=1 \text { or }\left(x_{n}, x_{n-1}, x_{k_{4}}\right)^{2 \gamma}=1 \text {, }
$$

both of which are impossible since $2 \gamma=2 q$ or $4 q$ and is not divisible by $r$.

Therefore, we have shown that if $G$ contains any nondeducible relation, we have a contradiction, and have thus proven the following theorem.

THEOREM 8. There are no groups of nilpotence class 3 which are $C N V$ groups with respect to a set of $n \geqq 3$ generators.

COROLlaRY. The only P-groups of nilpotence class 3 which are CNV groups must have presentations of the form (6.17), where

$$
\begin{gathered}
m=2^{u+v+w+1}, \quad p=2^{u+v}, \quad q=2^{u}, \quad \alpha=2^{u+v+w}, \quad u \geqq 1, \\
v, w \geqq 0, \quad v+w \geqq 1, \quad \beta=0 \text { iff } w \geqq 1, \\
\beta=2^{u+v-1} \text { if } w=0, \quad \delta=0 \text { or } 2^{u-1} \text { and } \delta=2^{u-1} \text { if } w \geqq 1 .
\end{gathered}
$$


Proof. We have shown that the only CNV groups of nilpotence class 3, must have presentations of form (6.17) and that if they are $P$-groups the $P$ must be 2 . Since $q \mid m, q$ is a power of 2 , say $q=2^{u}$. Since the group is not nilpotent of class $2, q \neq 1$, so $u \geqq 1$.

$q$ divides $p$ so $p=2^{u+v}, v \geqq 0$.

$p$ divides $\alpha=m / 2$ so $\alpha=2^{u+v+w}, w \geqq 0$ and then $m=2^{u+v+w+1}$ and $\beta=0$ or $p / 2=2^{u+v-1}$.

$q$ divides $\beta$, so if $\beta \neq 0$ then $v-1 \geqq 0$ so $v \geqq 1$.

$q$ divides $\alpha(\alpha-1) / 2$ but $(\alpha-1)$ is odd so $v+w-1 \geqq 0$ or $v+w \geqq 1$.

$p$ divides $\beta+\alpha(\alpha-1) / 2$.

If $w \geqq 1$ then $p \mid \alpha / 2$ so $p \mid \alpha(\alpha-1) / 2$ and $p \mid \beta$. But then $\beta=0$. Conversely if $\beta=0$, $p \mid \beta$ so $p \mid \alpha(\alpha-1) / 2$ so $p \mid \alpha / 2$ since $(\alpha-1)$ is odd and so $w \geqq 1$. I.e. $\beta=0$ if and only if $w \geqq 1$. Finally, $\delta=0$ or $q / 2=2^{u-1}$. If $w \geqq 1$ then $\beta=0$ so $\delta \neq 0$ so $\delta=2^{u-1}$. Putting this all together, we get the corollary.

THEOREM 9. Every CNV group of nilpotence class 3 has the quaternions as a quotient group.

Proof. Using the presentation given in the corollary map $x \rightarrow U, y \rightarrow V$ where

$$
Q=\left\langle U, V \mid U^{4}=(U, V, U)=(U, V, V)=1, U^{2}=(U, V)\right\rangle .
$$

This is easily seen to be a homomorphism.

7. Nilpotence class $\geqq 4$. On the basis of $\$ \S 5$ and 6 , we are led to conjecture that in general there are no nilpotent CNV groups with respect to sets of at least three generators, and that the only possible CNV groups that are $P$-groups occur for $P=2$.

Because of the possibility of the existence, in the general case, of relations about which we might not be aware, these questions seem to be more difficult.

\section{BIBLIOGRAPHY}

1. D. E. Cohen, Characteristic subgroups of some relatively free groups, J. London Math. Soc. 43 (1968), 445-451. MR 37 \#1450.

2. P. Hall, A contribution to the theory of groups of prime power order, Proc. London Math. Soc. (2) 36 (1933), 29-95.

3. F. Levi, Uber die Untergruppen der freien Gruppen. II, Math. Z. 37 (1933), 90-97.

4. W. Magnus, A. Karrass and D. Solitar, Combinatorial group theory: Presentations of groups in terms of generators and relations, Pure and Appl. Math., vol. 13, Interscience, New York, 1966. MR 34 \#7617.

5. B. H. Neumann, On characteristic subgroups of free groups, J. London Math. Soc. 43 (1968), 445-451.

6. H. Neumann, Varieties of groups, Ergebnisse der Mathematik und ihrer Grenzgebiete, Band 37, Springer-Verlag, New York, 1967. MR 35 \#6734.

7. J. Nielsen, Die Isomorphismengruppe der freien Gruppen, Math. Ann. 91 (1924), 169-209. 
8. J. Nielsen, Die Gruppe der dreidimensionalen Gittertransformationen, Danske Vid. Selsk. Mat.-Fys. Medd. 12 (1924), 1-29.

9. E. Rapaport, Note on Nielsen transformations, Proc. Amer. Math. Soc. 10 (1959), 228-235. MR 21 \#3477.

\section{TEMPLE UNIVERSITY,}

Philadelphia, Pennsylvania 19122 